\title{
On the Publication of the Special Issue on Flexible Optoelectronic Materials and Devices
}

Optoelectronic devices are no longer hard, rigid, and squared products. Recently, smartphones with foldable display screen have been launched successfully. Rollable TVs are now out there. Moreover, user-interactive, ultrathin electronic sensors can be attached onto or even be incorporated into the skin of a human body and deform conformably with human motion. All these fascinating development in the field may have perhaps stemmed from early imagination in the field of flexible electronics, when people, for the first time, realized that electronic devices can also be produced using $\pi$-conjugated polymers.

For celebrating the $20^{\text {th }}$ year, since Alan J. Heeger, Alan G. MacDiarmid, and Hideki Shirakawa won the Nobel Prize in Chemistry in 2000 for the discovery and development of conductive polymers, we prepared a special issue on "Flexible Optoelectronic Materials and Devices" in Macromolecular Research. The issue includes five invited articles from the young, leading researchers in the field of flexible polymer electronics. As Guest Editors for this special issue, we appreciate all the authors for sharing their fruitful results and all the reviewers for their excellent suggestions to the works. We believe that these efforts will altogether help deepening the understanding of our readers on $\pi$-conjugated macromolecules and expanding their imagination on what beyond now is possible from these materials.

Moon Sung Kang, Guest Editor Associate Professor, Chemical and Biomolecular Engineering Sogang University

Do Hwan Kim, Guest Editor Professor, Chemical Engineering Hanyang University
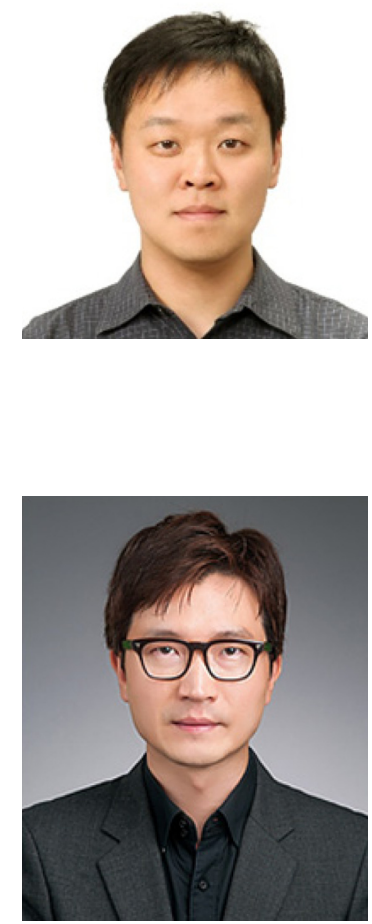